\begin{tabular}{|c|l|}
\hline Title & Long-time behavior of two-point functions of a quantum harmonic oscillator interacting with bosons \\
\hline Author(s) & A rai, A sao \\
\hline Citation & Journal of Mathematical Physics, 30(6), 1277-1288 \\
\hline https://doi.org/10.1063/1.528304 \\
\hline Issue Date & 1989-06 \\
\hline Doc URL & http://hdl.handle.net/2115/13678 \\
\hline Rights & Copyright $\odot$ 1989 A merican Institute of Physics \\
\hline Type & article \\
\hline File Information & jmp30-6.pdf \\
\hline
\end{tabular}

Instructions for use 


\title{
Long-time behavior of two-point functions of a quantum harmonic oscillator interacting with bosons
}

\author{
Asao Arai \\ Department of Mathematics, Hokkaido University, Sapporo 060, Japan
}

(Received 18 August 1988; accepted for publication 8 February 1989)

\begin{abstract}
A class of exactly soluble models of a one-dimensional quantum harmonic oscillator interacting with bosons moving in the $d$-dimensional space $\mathbf{R}^{d}$ is considered and the long-time behavior of the two-point function of the oscillator at zero temperature and at finite temperatures is analyzed. It is shown that the two-point functions decay with a power-law respectively as the time tends to infinity and that, in the case where the boson is massless, the two-point function at zero temperature decays faster than those at finite temperatures, while, in the case where the boson is massive, they decay with the same order. Further, the dependence of the decay order on $d$ as well as on the infrared behavior of the one-boson energy and the momentum cutoff function in the interaction Hamiltonian is clarified in each case.
\end{abstract}

\section{INTRODUCTION AND THE MAIN RESULTS}

In a previous paper,' we considered a general class of models of a one-dimensional quantum harmonic oscillator coupled to infinitely many bosons in the Hilbert space

$$
\mathscr{F}=L^{2}(\mathbf{R}) \otimes \mathscr{F}_{s}(\mathscr{H})
$$

where $\mathscr{F}_{s}(\mathscr{H})$ is the symmetric (Boson) Fock space over a complex Hilbert space $\mathscr{H}$ [e.g., Ref. 2 (§II.4)]. The unperturbed (free) Hamiltonian of each model in the class has the common form

$$
H_{0}=\omega_{0} a^{*} a \otimes I+I \otimes d \Gamma(h) .
$$

Here $a$ is the annihilation operator of the oscillator acting in $L^{2}(\mathbf{R}), d \Gamma(h)$ is the second quantization of a non-negative self-adjoint operator $h$ (the one-particle free Hamiltonian of the boson) [e.g., Ref. $3(\$ X .7)$ ], I denotes identity, and $\omega_{0}>0$ is a constant parameter denoting the circular frequency of the oscillator. The interaction part (perturbation) $H_{I}$ is given by a general form quadratic in the annihilation and creation operators for the bosons and the oscillator. (For the detailed form of $H_{I}$, see Ref. 1. But, in the present paper, we do not need it.) It was proved in Ref. 1 that, under some conditions, the total Hamiltonian

$$
H=H_{0}+H_{I}
$$

is unitarily equivalent to $d \Gamma(h)+E_{0}$ [acting in $\mathscr{F}_{s}(\mathscr{H})$ ] with a real constant $E_{0}$ and hence, in particular, all the possible embedded eigenvalues of $H_{0}$, except for the zero eigenvalue, disappear under the perturbation. Further, in the case $\mathscr{H}=L^{2}\left(\mathbf{R}^{d}\right)$, taking $H_{0}$ as

$$
H_{0}=\omega_{0} a^{*} a \otimes I+I \otimes d \Gamma\left(\omega^{r}\right)
$$

with a rotation invariant function $\omega$ on $\mathbf{R}^{d}$ and a parameter $r>0$ and changing the parameters in $H_{I}$, we showed that the class gives a unified description of standard models of a onedimensional quantum harmonic oscillator coupled to bosons, containing the following ones:

(M1) (The RWA oscillator; $r=1$ ) (e.g., Refs. 4-7)

$H=\omega_{0} a^{*} a \otimes I+I \otimes d \Gamma(\omega)+a \otimes b(\rho)^{*}+a^{*} \otimes b(\rho)$.

(M2) (The Schwabl-Thirring model; $r=1 / 2$ ) (e.g., Refs. 6, 8-13)

$$
H=\omega_{0} a^{*} a \otimes I+I \otimes d \Gamma\left(\omega^{1 / 2}\right)+q \otimes \phi(\rho) .
$$

$$
\begin{aligned}
& \text { (M3) }(r=1 / 2) \\
& H=\frac{1}{2} p^{2} \otimes I+I \otimes d \Gamma\left(\omega^{1 / 2}\right) \\
& +\left(\omega_{0}^{2} / 2\right)(q \otimes I-I \otimes \phi(\rho))^{2}-\frac{1}{2} \omega_{0} .
\end{aligned}
$$

Here $b(f), f \in L^{2}\left(\mathbf{R}^{d}\right)$, is the (smeared) boson annihilation operator acting in $\mathscr{F}_{s}\left(L^{2}\left(\mathbf{R}^{d}\right)\right), q \in \mathbf{R}$ denotes the position of the oscillator, $p=-i d / d q$,

$$
\phi(f)=(1 / \sqrt{2})\left\{b\left(\omega^{-1 / 4} f\right)^{*}+b\left(\omega^{-1 / 4} f\right)\right\},
$$

and $\rho$ is a cutoff function.

The model (M3) may be regarded as a simplified version of a three-dimensional quantum harmonic oscillator minimally coupled to a quantized radiation field with an ultraviolet cutoff (e.g., Ref. 14 and references therein).

In the present paper, taking $\mathscr{H}=L^{2}\left(\mathbf{R}^{d}\right)$ with $H_{0}$ given by (1.4), we investigate the long-time behavior of the two-point function at the zero temperature defined by

$$
W\left(t_{1}, t_{2}\right)=\left(\Omega, q\left(t_{1}\right) q\left(t_{2}\right) \Omega\right), \quad t_{1}, t_{2} \in \mathbf{R},
$$

and that at a finite temperature $\beta^{-1}>0$ given symbolically by

$$
W_{\beta}\left(t_{1}, t_{2}\right)=\frac{\operatorname{Tr}\left(e^{-\beta H} q\left(t_{1}\right) q\left(t_{2}\right)\right)}{\operatorname{Tr} e^{-\beta H}},
$$

where $q(t)$ is the time evolution of $q$ by the total Hamiltonian $H$ given by (1.3)

$$
q(t)=e^{i t H} q e^{-i t H}, \quad q \in \mathbf{R},
$$

$\Omega$ is the ground state of $H$, and Tr denotes the trace [strictly speaking, the rhs of (1.6) is defined as an infinite volume limit of a finite volume approximation of the quantum system under consideration]. As we shall see below, $W\left(t_{1}, t_{2}\right)$ [resp. $\left.W_{\beta}\left(t_{1}, t_{2}\right)\right]$ has a characteristic form common to all the models in the class. We are mainly interested in the following aspects:

(I) The dependence of the order of the time decay on the dimension $d, r$, and on the infrared property of the functions of $\omega$ and $\rho$ [i.e., the behavior of $\omega(\mathbf{k})$ and $\rho(\mathbf{k})$ as $\mathbf{k} \rightarrow 0$ ]

(II) The difference of the time decay between the case of finite temperatures $(0<\beta<\infty)$ and that of the zero temperature $(\beta=\infty)$.

(III) The difference of the time decay between the 
massless case $\left[\inf _{\mathbf{k}} \omega(\mathbf{k})=0\right]$ and the massive case $\left[\inf _{\mathbf{k}} \omega(\mathbf{k})>0\right]$.

We remark that we can not expect an exponential decay for the two-point functions, because we assume that $H$ is bounded from below to define them; this follows from a general theorem (Ref. 15, §7.3, Theorem 3.3). See also Appen$\operatorname{dix} \mathrm{C}$ in the present paper.

The problem of the long-time behavior of two-point functions (or correlation functions) of a quantum harmonic oscillator coupled to a heat bath (an infinite system of bosons) has recently been discussed in the statistical physics literature (e.g., Refs. 6, 12, 16, and 17; cf. also Ref. 10 for a field theoretical discussion) and some partial results have been obtained with some concrete models [mainly those related to the models (M1) and (M2)]. In these works, however, the authors make an ad hoc assumption on the spectral density of the correlation function or on the memory kernel in the Langevin equation. This procedure formally corresponds to taking a special form for the cutoff function $\rho$ in our Hamiltonian formalism. In the present paper, we do not make such an $a d$ hoc assumption and consider the models as generally as possible. Accordingly, our results include as special cases the partial results on the long-time behavior mentioned above and generalize on them. At the end of this section, after stating our results, we shall give a more detailed comparison of our results with those obtained in the other works.

We now proceed to describe the two-point functions in our models.

Let $\omega_{1}$ be a non-negative, continuously differentiable, and monotone increasing function on $(0, \infty)$ such that $\omega_{1}(x) \rightarrow \infty$ as $x \rightarrow \infty$ with the derivative $\omega_{1}^{\prime}(x)>0$ for all $x>0$. We set

$$
m=\inf _{x>0} \omega_{1}(x) \geqslant 0 .
$$

The rotation invariant function $\omega$ on $\mathbf{R}^{d}$ is defined by

$$
\omega(\mathbf{k})=\omega_{1}(|\mathbf{k}|), \quad \mathbf{k} \in \mathbf{R}^{d} .
$$

The cutoff function $\rho$ is a real-valued continuous function in $L^{2}\left(\mathbf{R}^{d}\right)$ such that

$$
\int_{\mathbf{R}^{i}} \frac{\rho(\mathbf{k})^{2}}{\omega(\mathbf{k})} d \mathbf{k}<\infty
$$

and

$$
\int_{\mathbf{R}^{d}} \frac{\rho(\mathbf{k})^{2}}{|m-\omega(\mathbf{k})|} d \mathbf{k}<\infty .
$$

Remark: If $m>0$ (the massive case), then (1.10) is automatically satisfied because of $\rho \in L^{2}\left(\mathbf{R}^{d}\right)$ [more generally, we have $\omega^{-\lambda} \rho \in L^{2}\left(\mathbf{R}^{d}\right)$ for all $\left.\lambda>0\right]$, but, in the case $m=0$ (the massless case), this is not true.

For $j=0,1$, we introduce the function $\Phi_{\rho}^{(j)}(z)$ by

$$
\Phi_{\rho}^{(j)}(z)=\int_{\mathbf{R}^{i}} \frac{\omega(\mathbf{k})^{j} \rho(\mathbf{k})^{2}}{z-\omega(\mathbf{k})} d \mathbf{k},
$$

which is analytic in the cut plane

$$
\mathbf{C}_{m}=\mathbf{C} \backslash[m, \infty) \text {. }
$$

Let

$$
D^{(j)}(z)=a_{0}^{(j)}-a_{1}^{(j)} z+\delta_{1, j} \int \rho(\mathbf{k})^{2} d \mathbf{k}+\Phi_{\rho}^{(j)}(z)
$$

where $a_{i}^{(j)}, i, j=0,1$, are real constants with $a_{1}^{(j)}>0, j=0,1$, and $\delta_{1, j}$ is the Kronecker delta function. We assume that

$$
\begin{aligned}
d_{m}^{(j)} \equiv & a_{0}^{(j)}-a_{1}^{(j)} m+\delta_{1, j} \int \rho(\mathbf{k})^{2} d \mathbf{k} \\
& +\Phi_{\rho}^{(j)}(m)>0 .
\end{aligned}
$$

Then it is easy to see that

$$
D^{(j)}(z) \neq 0, \quad z \in \mathrm{C}_{m} \text {. }
$$

Remark: By renormalizing the parameter $a_{0}^{(j)}$ as

$$
\begin{gathered}
\tilde{a}_{0}^{(j)}=a_{0}^{(j)}+a_{1}^{(j)} m-\delta_{1, j} \int \rho(\mathbf{k})^{2} d \mathbf{k}-\Phi_{\rho}^{(j)}(m), \\
a_{0}^{(j)}>0,
\end{gathered}
$$

$d_{m}^{(j)}$ can be made positive.

For technical reasons, we assume the following:

$$
\begin{gathered}
\text { (AI) (a) } \sup _{\substack{\epsilon>0 \\
x \in[m, \infty) .}}\left|\Phi_{\rho}^{(j)}(x-i \epsilon)\right|<\infty, \\
\text { (b) } \inf _{\substack{\epsilon>0 \\
x \in[m, \infty)}}\left|D^{(j)}(x-i \epsilon)\right|>0 .
\end{gathered}
$$

For sufficient conditions for (AI) to hold, see Appendices A and $B$. $x>0$,

It follows from (AI) (a) that, for all sufficiently large

$$
\left|D^{(j)}(x-i \epsilon)\right| \geqslant c x
$$

with a constant $c>0$ independent of $\epsilon$.

One can show using the theory of the Hilbert transform (e.g., Ref. 18) that the limits

$$
D_{ \pm}^{(j)}(x) \equiv \lim _{\epsilon \uparrow 0} D^{(j)}(x \pm i \epsilon), \quad j=0,1
$$

exist for a.e. $x \epsilon(m, \infty)$, which, by assumption $(\mathrm{AI})(\mathrm{b})$, cannot be zero.

We are now ready to give an explicit form of $W\left(t_{1}, t_{2}\right)$ [ resp. $W_{\beta}\left(t_{1}, t_{2}\right)$ ] (up to a constant multiple) which follows from Ref. 1:

$$
\begin{aligned}
& W\left(t_{1}, t_{2}\right)=W\left(t_{1}-t_{2}\right), \\
& W_{\beta}\left(t_{1}, t_{2}\right)=W_{\beta}\left(t_{1}-t_{2}\right),
\end{aligned}
$$

with

$$
\begin{aligned}
W(t)= & \int_{\mathbf{R}^{d}} \frac{\rho(\mathbf{k})^{2} e^{-i t \omega(\mathbf{k})^{r}}}{\omega(\mathbf{k})^{2 r \alpha}\left|D_{+}^{(j)}(\omega(\mathbf{k}))\right|^{2}} d \mathbf{k}, \\
& j=0,1,
\end{aligned}
$$

and

$$
\begin{aligned}
W_{\beta}(t)= & \int_{\mathbf{R}^{d}} \frac{\rho(\mathbf{k})^{2}\left(e^{(\beta-i t) \omega(\mathbf{k})^{\prime}}+e^{i t \omega(\mathbf{k})^{r}}\right)}{\omega(\mathbf{k})^{2 r \alpha}\left|D_{+}^{(j)}(\omega(\mathbf{k}))\right|^{2}\left(e^{\beta \omega(\mathbf{k})^{r}}-1\right)} d \mathbf{k} \\
& j=0,1
\end{aligned}
$$

Here $r>0$ is the parameter in $H_{0}$ given by (1.4) and $\alpha \in \mathbf{R}$ is a parameter appearing in $b\left(\omega^{-r \alpha} \rho\right)$ and $b\left(\omega^{-r \alpha} \rho\right)^{*}$ in the interaction $H_{I}$, and $j$ depends on the form of $H_{l}$; for example, in the model (M1) [resp. (M2), (M3)], we have $(r, \alpha$, $j)=(1,0,0)\left[\operatorname{resp} .\left(\frac{1}{2}, \frac{1}{2}, 0\right),\left(\frac{1}{2}, \frac{1}{2}, 1\right)\right]$. Of course, the con- 
stants $a_{0}^{(j)}$ and $a_{1}^{(j)}$ also change according to models : In the model (M1) [resp. (M2), (M3)], we have $a_{0}^{(0)}=\omega_{0}$ and $a_{1}^{(0)}=1\left[\right.$ resp. $\left.a_{0}^{(0)}=\omega_{0}^{2} a_{1}^{(0)}, a_{0}^{(1)}=\omega_{0}^{2} a_{1}^{(1)}\right]$. In deriving (1.21) [resp. (1.22)] with the case $m=0$, an additional assumption that $\rho^{2} /\left(\omega^{2 r \alpha}\left|D_{+}^{(j)}(\omega)\right|^{2}\right) \quad$ [resp. $\left.\rho^{2} / \omega^{2 r \alpha+1}\left|D_{+}^{(j)}(\omega)\right|^{2}\right]$ is integrable near the origin is made. Note that, for all $t \in \mathbf{R}, W_{\beta}(t) \rightarrow W(t)$ as $\beta \rightarrow \infty$ (the zerotemperature limit).

To make the present paper self-contained, we take formulas (1.19)-(1.22) as the starting point. Therefore the reader is not required to have any detailed knowledge in $R e f$. 1.

We shall denote by $\eta(x)$ the inverse function of $\omega_{1}(x)$,

$\omega_{1}(\eta(x))=x$

for $x>m$. By the inverse function theorem, $\eta(x)$ is differentiable and monotone increasing in $(m, \infty)$ with

$$
\begin{aligned}
& \lim _{x \backslash m} \eta(x)=0, \quad \eta^{\prime}(x)=\left[\omega_{1}^{\prime}(\eta(x))\right]^{-1}, \\
& x \in(m, \infty) .
\end{aligned}
$$

For a measurable function $f$ on $\mathbf{R}^{d}$, we define the function $[f]$ on $[0, \infty)$ by

$$
[f](x)=\int_{S^{d-1}} d S(\theta) f(x \theta), \quad x \in[0, \infty),
$$

where $S^{d-1}$ is the $d-1$ sphere and $d S$ is the surface integral measure on $S^{d-1}$. Let

$$
I^{(j)}(x)=\eta^{\prime}(x) \eta(x)^{d-1} x^{j}\left[\rho^{2}\right](\eta(x)), \quad x \in(m, \infty) .
$$

Since $\rho$ is continuous by assumption, it follows that $I^{(j)}(x)$ is continuous in $x \in(m, \infty)$.

As another technical assumption, we take the following:

(AII) There exists a constant $\theta \in(0, \pi / 2)$ such that the function $I^{(j)}(x)$ has an analytic continuation $I^{(j)}(z)$ onto the domain

$$
\mathbf{D}_{m, \theta}=\{z \in \mathbf{C} \mid \operatorname{Re} z>m,-\theta<\arg z<0\}
$$

with the following properties:

(a) $\lim _{\epsilon 10} I^{(j)}(x-i \epsilon)=I(x), \quad x \in(m, \infty)$.

(b) $\left|I^{(j)}(z)\right| \leqslant$ const $|z|^{-q_{j}}$

for all sufficiently large $|z|\left(z \in \mathbf{D}_{m, \theta}\right)$ with a constant $q_{j} \geqslant 0$.

(c) $\lim _{\substack{z \rightarrow 0 \\ z \in \mathbf{D}_{0, \theta}}} \frac{I^{(j)}(m+z)}{z^{p^{(m)}}}=A_{m}^{(j)}$

with constants $A_{m}^{(j)} \neq 0$ and $p_{j}(m) \geqslant 0$.

(d) For all sufficiently small $\epsilon_{0}>0$,

$$
\inf _{\substack{0<\epsilon<\epsilon_{i} \\ x \in[m, \infty)}}\left|D^{(j)}(x-i \epsilon)-2 i \pi I^{(j)}(x-i \epsilon)\right|>0 .
$$

Remark: As is seen from the definition of $I^{(j)}(x), p_{j}(m)$ is determined by the dimension $d$ and the infrared property of $\omega_{1}, \omega_{1}^{\prime}$, and $\rho$. See Sec. IV for an example.

In order to investigate the decay property of $W(t)$ and $W_{\beta}(t)$ in a unified way, we introduce the following more general function:

$$
W(t, f)=\int_{\mathbf{R}^{d}} \frac{f(\omega(\mathbf{k})) \rho(\mathbf{k})^{2} e^{-i t \omega(\mathbf{k})^{\prime}}}{\omega(\mathbf{k})^{2 r \alpha}\left|D_{+}^{(j)}(\omega(\mathbf{k}))\right|^{2}} d \mathbf{k}
$$

Here $f$ is any meromorphic function in $\mathbf{D}_{m, \theta}$ with the following properties:

(f I) The number of poles of $f(z)$ is finite.

(f II) The limit

$$
\lim _{\epsilon 10} f(x-i \epsilon) \equiv f(x)
$$

exists for a.e. $x \in(m, \infty)$.

(f III) $\sup \left\{|f(z)|\left|z \in \mathbf{D}_{m, \theta} \cup(m, \infty),\right| z \mid \geqslant r_{0}\right\}<\infty$ with a constant $r_{0}>0$.

$$
\text { (f IV) } \lim _{\substack{z \rightarrow 0 \\ z \in \mathbf{D}_{0, \theta}}} z^{\alpha_{m}(n)} f(m+z)=f_{m}
$$

with constants $\alpha_{m}(f) \in \mathbf{R}$ and $f_{m} \neq 0$.

We now state the main results. We mean by

$$
g(t) \underset{t \rightarrow \infty}{\sim} h(t)
$$

that

$$
\lim _{t \rightarrow \infty} \frac{g(t)}{h(t)}=1 .
$$

Let

$$
B_{m}^{(j)}=\left(D^{(j)}(m)-2 i \pi \delta_{0, p_{j}(m)} A_{m}^{(j)}\right) D^{(j)}(m) .
$$

Theorem 1.1: Suppose that $2 r \alpha+j+q_{j}+1>0$.

(a) Let $m=0$ and suppose that

$$
\mu_{j} \equiv \frac{p_{j}(0)-\alpha_{0}(f)-2 r \alpha-j+1}{r}>0 .
$$

Then

$$
W(t, f) \underset{i \rightarrow \infty}{\sim} \frac{A_{0}^{(j)} f_{0} e^{-i \pi \mu_{j} / 2} \Gamma\left(\mu_{j}\right)}{r B_{0}^{(j)}} t^{-\mu_{j}},
$$

where $\Gamma(z)$ is the gamma function.

(b) Let $m>0$ and

$$
v_{j}=p_{j}(m)-\alpha_{m}(f)+1>0 .
$$

Then

$$
W(t, f) \underset{t \rightarrow \infty}{\sim} \frac{A_{m}^{(j)} f_{m} e^{-i \pi v_{j} / 2} \Gamma\left(v_{j}\right) e^{-i t m^{r}}}{r^{v_{j}} m^{2 m+j+(r-1) v_{j}} B_{m}^{(j)}} t^{-v_{j}} .
$$

Remark: Under the assumption of Theorem 1.1 in each case $m=0$ or $m>0$, the integral of the rhs of (1.28) is absolutely convergent.

As corollaries of Theorem 1.1, we can derive the asymptotic behavior of $W(t)$ and $W_{\beta}(t)$ as $t \rightarrow \infty$.

Theorem 1.2 (the massless case): Let $m=0$ and $2 r \alpha+j+q_{j}+1>0$.

$$
\begin{aligned}
& \text { (a) Suppose that } \\
& \lambda_{j} \equiv \frac{p_{j}(0)-2 r \alpha-j+1}{r}>0 .
\end{aligned}
$$

Then

$$
W(t) \underset{t \rightarrow \infty}{\sim} \frac{A_{0}^{(j)} e^{-i \pi \lambda_{j} / 2} \Gamma\left(\lambda_{j}\right)}{r B_{0}^{(j)}} t^{-\lambda_{j}} .
$$

(b) Suppose that $\lambda_{j}>1$. Then 
$W_{\beta}(t) \underset{t \rightarrow \infty}{\sim} 2 \operatorname{Re}\left\{\frac{A_{0}^{(j)} e^{-i \pi\left(\lambda_{j}-1\right) / 2} \Gamma\left(\lambda_{j}-1\right)}{\beta r B_{0}^{(j)}}\right\} t^{-\lambda_{j}+1}$.

Proof: (a) We have

$$
W(t)=W(t, f \equiv 1) .
$$

On the other hand, the function $f(z) \equiv 1$ obviously possesses properties (f I) and (fII) with $\alpha_{0}(f)=0$ and $f_{0}=1$. Therefore, in this case, we have $\mu_{j}=\lambda_{j}$. Thus (1.31) yields (1.35).

\section{(b) Let}

$$
f(z)=e^{\beta z^{r}} /\left(e^{\beta z^{\prime}}-1\right)
$$

and

$$
g(z)=1 /\left(e^{\beta \bar{z}^{r}}-1\right) .
$$

Then we have

$$
W_{B}(t)=W(t, f)+\overline{W(t, \bar{g})} .
$$

It is easy to see that each of $f$ and $\bar{g}$ satisfies (f $\mathrm{I}$ ) and (f II) with $\alpha_{0}(f)=\alpha_{0}(\bar{g})=r$ and $f_{0}=\bar{g}_{0}=1 / \beta$. Hence we have $\mu_{j}=\lambda_{j}-1$. Thus we get (1.36) from (1.31).

Theorem 1.3 (the massive case): Let $m>0$ and $2 r a+j+q_{j}+1>0$. Then

$$
\text { (a) } \begin{aligned}
& W(t) \underset{t \rightarrow \infty}{\sim} \frac{A_{m}^{(j)} e^{-i m\left(p_{j}(m)+1\right) / 2} \Gamma\left(p_{j}(m)+1\right)}{r^{p_{j}(m)+1} m^{2 r \alpha+j+(r-1)\left(p_{j}(m)+1\right)} B_{m}^{(j)}} \\
& \times e^{-i t m^{\prime} t} p^{-p_{j}(m)-1} .
\end{aligned}
$$

(b) $W_{\beta}(t) \underset{t \rightarrow \infty}{\sim} \frac{A_{m}^{(j)} \Gamma\left(p_{j}(m)+1\right)}{\left(e^{\beta m^{r}}-1\right) r^{p_{j}(m)+1} m^{2 r \alpha+j+(r-1)\left(p_{j}(m)+1\right)}}$

$$
\begin{aligned}
& \times\left\{\frac{e^{(\beta-i t) m^{r}-i m\left(p_{j}(m)+1 / / 2\right.}}{B_{m}^{(j)}}\right. \\
& \left.+\frac{e^{i t m^{r}+i m\left(p_{j}(m)+1\right) / 2}}{\overline{B_{m}^{(j)}}}\right\} t^{-p_{J}(m)-1} .
\end{aligned}
$$

\section{Proof: Similar to the proof of theorem 1.2.}

Theorems 1.2 and 1.3 show that the two-point functions decay with a power-law respectively as $t \rightarrow \infty$. In both cases $m=0$ and $m>0$, the order of the decay increases as $p_{j}(m)$ does. On the other hand, the dependence of the decay order on the dimension $d$ and the infrared behavior of $\omega$ and $\rho$ comes only from $p_{j}(m)$ [see (1.26) and (AII) (c) ]. In particular, it follows from $(1.26)$ that $p_{j}(m)$ is monotone increasing in $d$. Therefore the higher $d$ becomes, the faster the two-point functions decay.

In the massless case, Theorem 1.2 shows that $W(t)$ decays faster than $W_{B}(t)$. On the other hand, in the massive case, the order of the decay of $W(t)$ coincides with that of $W_{\beta}(t)$. We note also that, in the massive case, the oscillating factors $\exp \left( \pm \mathrm{itm}^{r}\right)$ appear in the asymptotic behavior of the two-point functions.

Remark: We have assumed $d_{m}^{(j)}>0[(1.15)]$ to obtain Theorems 1.1-1.3. In fact, in the case $d_{m}^{(j)}<0, D^{(j)}(z)$ has a simple unique zero $v_{0} \in(-\infty, m)$ and the oscillating terms proportional to $\exp \left( \pm i v_{0} t\right)$ appear in the two-point functions. This corresponds to the nondisappearence of embed- ded eigenvalues of $H_{0}$ under the perturbation (in other words, the oscillator mode persists under the perturbation) (cf. Ref. 7).

We now try to compare in some detail our results with those on the long-time behavior obtained in the other works. ${ }^{6,12,16,17}$

In Ref. 16, a phenomenological model based on an averaged Langevin equation rather than on a Hamiltonian was presented; the correlation function at the inverse temperature $\beta$ is given as

$$
\langle q(t) q(0)\rangle=\int_{-\infty}^{\infty} \frac{d \omega}{\pi} \chi^{\prime \prime}(\omega) \frac{\hbar e^{-i \omega t}}{1-e^{-\beta \hbar \omega}},
$$

where $\chi^{\prime \prime}(\omega)$ is the imaginary part of the Fourier transform of the response function,

$$
\chi^{\prime \prime}(\omega)=\frac{1}{M} \cdot \frac{\gamma \omega}{\left(\omega^{2}-\omega_{0}^{2}\right)^{2}+\gamma^{2} \omega^{2}}
$$

with positive constants $\gamma$ (the damping constant) and $M$ (the mass of the oscillator) and $\hbar$ is the Planck constant divided by $2 \pi$. They showed that, at the zero temperature $\beta \rightarrow \infty$, the real part $S(t)$ (the symmetrized correlation function) decays as

$$
S(t) \underset{t \rightarrow \infty}{\sim}-\frac{\hbar \gamma}{\pi M \omega_{0}^{4}} \frac{1}{t^{2}} .
$$

We can show that this result is a special case of our results: in fact, at the zero temperature $(\beta \rightarrow \infty)$, one has from (1.39)

$$
\langle q(t) q(0)\rangle=\int_{0}^{\infty} \frac{d \omega}{\pi} \chi^{\prime \prime}(\omega) \hbar e^{-i \omega t} .
$$

On the other hand, the function $W(t)$ given by $(1.21)$ is written as

$$
W(t)=\int_{m}^{\infty} w(x) e^{-i t x^{r}} d x
$$

with

$$
w(x)=\frac{I^{(j)}(x)}{x^{2 r d \alpha+j}\left|D_{x}^{(j)}(x)\right|^{2}} .
$$

Therefore, by considering the case with $m=0$ and $r=1$ and by setting formally

$$
w(x)=(\hbar / \pi) \chi^{\prime \prime}(x)
$$

we have at the zero temperature

$$
W(t)=\langle q(t) q(0)\rangle
$$

Since $\chi^{\prime \prime}(x) \sim \gamma x /\left(M \omega_{0}^{4}\right)$ as $x \rightarrow 0$, we have

$$
A_{0}^{(j)} / B_{0}^{(j)}=\hbar \gamma / \pi M \omega_{0}^{4}, \quad \lambda_{j}=2 .
$$

Then, (1.41) follows from (1.35). On the other hand, at finite temperatures $\beta^{-1}>0,\langle q(t) q(0)\rangle$ is not of the form $W_{\beta}(t)$ given by (1.22), because the frequency spectrum of $\langle q(t) q(0)\rangle$ is equal to the whole real line $\mathbf{R}$ as is seen from (1.39). [The frequency (energy) spectrum of our models is equal to $[m, \infty)$.] This is a big difference between the model under consideration and ours. They also showed that, at finite temperatures, $\langle q(t) q(0)\rangle$ decays exponentially. We remark that, if one modifies the correlation function as

$$
\langle q(t) q(0)\rangle_{+}=\int_{0}^{\infty} \frac{d \omega}{\pi} \chi^{\prime \prime}(\omega) \frac{\hbar e^{-i \omega t}}{1-e^{-\beta \hbar \omega}}
$$


or

$$
\langle q(t) q(0)\rangle_{-}=\int_{-\infty}^{0} \frac{d \omega}{\pi} \chi^{\prime \prime}(\omega) \frac{\hbar e^{-i \omega t}}{1-e^{-\beta \hbar \omega}},
$$

then one gets

$$
\begin{aligned}
& \langle q(t) q(0)\rangle_{+} \underset{t \rightarrow \infty}{\sim} \frac{(-i) \gamma}{\pi M \omega_{0}^{4}} \cdot \frac{1}{t}, \\
& \langle q(t) q(0)\rangle_{-} \underset{t \rightarrow \infty}{\sim} \frac{i \gamma}{\pi M \omega_{0}^{4} \beta} \cdot \frac{1}{t} .
\end{aligned}
$$

Namely, $\langle q(t) q(0)\rangle_{ \pm}$have a power-law decay. This follows from an application of Theorem 2.1(a) in Sec. II.

In Ref. 17, a generalized version of the model in Ref. 16 was considered and a more detailed analysis on the long-time behavior of the correlation functions was done. As in the model in Ref. 16, the correlation function at zero temperature is a special case of our model and the result on the longtime behavior coincides with ours, at least, up to $t^{-2}$ order. As for the case at finite temperatures, the same remark as that concerning the model in Ref. 16 applies.

In Ref. 12, they start from a discrete version of the model (M2) with respect to the boson degrees of freedom and derive the dynamical equation for the position operator of the oscillator. Then, they make an ad hoc assumption for the memory kernel so that the symmetrized autocorrelation function of the fluctuating force per unit mass $\Phi_{T}(t)$ and the symmetrized autocorrelation function of the particle velocity $C_{v v}(t)$ take the form

$$
\Phi_{T}(t)=\frac{\hbar \gamma^{2}}{M \tau_{R}} \int_{-\infty}^{\infty} \frac{d \omega}{2 \pi} \cdot \frac{\omega}{\omega^{2}+\gamma^{2}} \operatorname{coth} \frac{\beta \hbar \omega}{2} e^{i \omega t}
$$

and

$$
\begin{aligned}
C_{v v}(t)= & \frac{\hbar \gamma^{2}}{M \tau_{R}} \int_{-\infty}^{\infty} \frac{d \omega}{2 \pi} \frac{\omega^{3}}{\omega^{2}+\gamma^{2}} \\
& \times \frac{1}{\left|\omega^{2}+\left(\gamma / \tau_{R}\right)[i \omega /(\gamma-i \omega)]\right|^{2}} \\
& \times \operatorname{coth} \frac{\beta \hbar \omega}{2} e^{i \omega t},
\end{aligned}
$$

respectively, where $\tau_{R}>0$ is a constant. They showed that, at finite temperatures, $\Phi_{T}(t)$ and $C_{v v}(t)$ decay exponentially as $t \rightarrow \infty$ and, only in an intermediate time region, display a long time tail as const $t^{-2}$. These results do not coincide with our results. This is due to the fact that, in the present case, the frequency spectrum runs from $-\infty$ to $+\infty$ as is seen from (1.45) and (1.46), which is an effect of the symmetrization. Let $\Phi_{T}^{(+)}(t)$ and $C_{v v}^{(+)}(t)$ be the functions defined by the rhs of (1.45) and (1.46) with the integral interval replaced by $[0, \infty)$, respectively. Then, we see that $\Phi_{T}^{(+)}(t)$ and $C_{v v}^{(+)}(t)$ display a power-law decay as

$$
\Phi_{r}^{(+)}(t) \underset{t \rightarrow \infty}{\sim} \frac{i}{\pi M \tau_{R} \beta} \cdot \frac{1}{t}
$$

and

$$
C_{v v}^{(+)}(t) \underset{t \rightarrow \infty}{\sim} \frac{i \tau_{R}}{\pi M \beta} \cdot \frac{1}{t}
$$

At zero temperature, we have

$$
\begin{aligned}
\Phi_{T}(t)= & \frac{\hbar \gamma^{2}}{M \tau_{R} \pi} \operatorname{Re}\left\{\int_{0}^{\infty} d \omega \frac{\omega}{\omega^{2}+\gamma^{2}} e^{i \omega t}\right\} \\
C_{v v}(t)= & \frac{\hbar \gamma^{2}}{M \tau_{R} \pi} \operatorname{Re}\left\{\int_{0}^{\infty} d \omega \frac{\omega^{3}}{\omega^{2}+\gamma^{2}}\right. \\
& \left.\times \frac{e^{i \omega t}}{\left|\omega^{2}+\left(\gamma / \tau_{R}\right)[i \omega /(\gamma-i \omega)]\right|^{2}}\right\} .
\end{aligned}
$$

By integration by parts and applying Theorem 2.1(a), we get

$$
\begin{aligned}
& \Phi_{T}(t) \underset{t \rightarrow \infty}{\sim}-\frac{\hbar}{M \tau_{R} \pi} \cdot \frac{1}{t^{2}}, \\
& C_{V V}(t) \underset{t \rightarrow \infty}{\sim}-\frac{\hbar \tau_{R}}{M \pi \gamma^{2}} \cdot \frac{1}{t^{2}} .
\end{aligned}
$$

These results are not given in Ref. 12 .

In Ref. 6, the authors start from the models (M1) and (M2) with a discrete boson degrees of freedom and calculate the momentum autocorrelation function $C(t)$ of the oscillator

$$
C(t)=C_{0}(t)+C_{1}(t)
$$

with

$$
\begin{aligned}
& C_{1}(t)=\int_{0}^{\infty} \frac{1}{e^{\beta \lambda \lambda}-1} G(\lambda) \cos (\lambda t) d \lambda, \\
& C_{0}(t)=\frac{1}{2} \int_{0}^{\infty} G(\lambda) e^{-i \lambda t} d \lambda,
\end{aligned}
$$

where $G(\lambda)$ is a function depending on the choice of the models (M1) and (M2). The exact form of $G(\lambda)$ was not given and, by assuming that $G(\lambda) \sim \lambda^{m}$, they showed that, at low temperatures, $C_{1}(t)$ decays with a power law as $t \rightarrow \infty$. However, analysis on the long-time behavior of $C_{1}(t)$ at nonlow temperatures and of $C_{0}(t)$ (the correlation function at zero temperature) was not given. We note that (1.49) with (1.50) and (1.51) is exactly of the same form as $W_{B}(t)$ given by (1.22) with $m=0$. This is easily seen by change of variable. Thus we can apply our results and solve the problem left in Ref. 6. One can easily check that our general results yield as a special case the partial result on the longtime behavior of $C_{1}(t)$ obtained in Ref. 6 .

The rest of the present paper is organized as follows: In Sec. II, we establish a limit theorem on an integral of Fourier's type. In Sec. III, applying the limit theorem, we prove Theorem 1.1. In Sec. IV, we discuss an example of $\omega$. In Appendices $A$ and $B$, sufficient conditions for (AI) to hold are given. In Appendix C, a necessary condition for exponential decay of Fourier transforms is given.

\section{A LIMIT THEOREM}

In this section, we prove a general limit theorem concerning an integral of Fourier's type.

Let $g$ be a measurable function in $L^{1}((m, \infty), d x)$. We are concerned with the asymptotic behavior of the function

$$
G(t)=\int_{m}^{\infty} g(x) e^{-i t x^{\prime}} d x \quad(t>0)
$$

as $t \rightarrow \infty$, where $m \geqslant 0$ and $r>0$ are constants. We assume the following: 
$(A-g)_{m}$ There exists a meromorphic function $g(z)$ in $\mathbf{D}_{m, \theta}$ with the following properties:

(a) $\lim _{\epsilon \downarrow 0} g(x-i \epsilon)=g(x)$, a.e. $x \in(m, \infty)$,

(b) For all sufficiently small $\epsilon>0$ and every $a, b$ $\in(m, \infty)(a<b)$,

$$
\sup _{a<x<b}|g(x-i \epsilon)| \leqslant C_{a, b}
$$

with a constant $C_{a, b}>0$ independent of $\epsilon$.

(c) For all sufficiently large $|z|\left(z \in \mathbf{D}_{m, \theta}\right)$

$|g(z)| \leqslant C /|z|^{q}$

with constants $C>0$ and $q>1$.

(d) $g(z)$ has no poles in $\left\{z \in \mathbf{D}_{m, \theta}|| z-m \mid<\epsilon_{0}\right\}$ with a constant $\epsilon_{0}>0$ and the limit

$$
\lim _{z \rightarrow 0} \frac{g(m+z)}{z^{\mu_{m}}} \equiv g_{m} \neq 0
$$

exists with constants $g_{m}$ and $\mu_{m}>-1$.

Under the assumption $(A-g)_{m}$, we have the following theorem.

Theorem 2.1: (a) Let $m=0$. Then

$G(t) \underset{t \rightarrow \infty}{\sim} \frac{g_{0} e^{-i \pi\left(\mu_{1}+1\right) / 2 r} \Gamma\left(\left(\mu_{0}+1\right) / r\right)}{r} t-\left(\mu_{10}+1\right) / r$.

(b) Let $m>0$. Then

$$
\begin{aligned}
G(t) \underset{t \rightarrow \infty}{\sim} & \frac{g_{m} m^{(1-r)\left(\mu_{m}+1\right)} e^{-i t\left[m^{r}+\left(\mu_{m}+1\right) / 2\right]} \Gamma\left(\mu_{m}+1\right)}{r^{\mu_{m}+1}} \\
& \times t^{-\left(\mu_{m}+1\right)} .
\end{aligned}
$$

Proof: We first consider the case $m=0$. Let

$$
h_{t}(z)=e^{-i t z r} g(z), \quad \mathrm{t}>0 .
$$

Then $h_{t}(z)$ is meromorphic in $\mathbf{D}_{0, \theta}$. Let $\epsilon>0, \delta>0$ be sufficiently small, $L>0$ be sufficiently large, and $0<\theta_{0}<\theta$. Let $\Gamma\left(\epsilon, \delta, L, \theta_{0}\right)$ be the curve in $\mathbf{D}_{0, \theta}$ given by

$$
\begin{aligned}
\Gamma\left(\epsilon, \delta, L, \theta_{0}\right)= & \{x-i \epsilon \mid \delta \leqslant x \leqslant L\} \\
& \cup\left\{\delta-i \epsilon+s e^{-i \theta_{11}} \mid 0 \leqslant s \leqslant L\right\} \\
& \cup\left\{\delta-i \epsilon+L e^{i u} \mid-\theta_{0} \leqslant u \leqslant 0\right\}
\end{aligned}
$$

with the anticlockwise orientation and $\mathrm{D}\left(\epsilon, \delta, L, \theta_{0}\right)$ be the interior domain of $\Gamma\left(\epsilon, \delta, L, \theta_{0}\right)$. Let $\left\{a_{k}\right\}_{k=1}^{N}$ be poles in $\mathrm{D}_{0, \theta_{0}}\left[N\right.$ is finite by $\left.(\mathrm{Ag})_{0}(\mathrm{~b})-(\mathrm{d})\right]$. Then, by applying the Cauchy integral theorem to the integral of $h_{t}(z)$ along $\Gamma\left(\epsilon, \delta, L, \theta_{0}\right)$, we get

$$
\begin{aligned}
\int_{\delta}^{L} h_{t}(x-i \epsilon) d x= & \int_{0}^{L} h_{t}\left(\delta-i \epsilon+s e^{-i \theta_{i n}}\right) e^{-i \theta_{t}} d s \\
& +\int_{-\theta_{t},}^{0} h_{t}\left(\delta-i \epsilon+L e^{i u}\right) i L e^{i u} d u \\
& -2 \pi i \sum_{a_{k} \in \mathbf{D}\left(\epsilon, \delta, L, \theta_{11}\right)} \operatorname{Res}\left(h_{t}(z), a_{k}\right)
\end{aligned}
$$

where $\operatorname{Res}\left(h_{t}(z), a_{k}\right)$ denotes the residue of $h_{t}(z)$ at $z=a_{k}$ [we take $\Gamma\left(\epsilon, \delta, L, \theta_{0}\right)$ so that $a_{k} \notin \Gamma(\epsilon, \delta, L, \theta)$ for all $k$ ]. We take $\theta_{0}$ to satisfy $0<r \theta_{0}<\pi / 2$. Then we have by $(A-g)_{0}-(\mathrm{c})$

$$
\left|h_{t}\left(\delta-i \epsilon+L e^{i u}\right)\right| \leqslant C / L^{q}
$$

for all sufficiently large $L$ with a constant $C>0$ independent of $L$. Hence we get

$$
\lim _{L \rightarrow \infty} \int_{-\theta_{0}}^{0} h_{t}\left(\delta-i \epsilon+L e^{i u}\right) i L e^{i u} d u=0 .
$$

For $s \geqslant s_{0}>0$, we have by $(\mathrm{Ag})_{0}-(\mathrm{c})$

$$
\left|h_{t}\left(\delta-i \epsilon+s e^{-i \theta_{i}}\right)\right| \leqslant a e^{-b s^{\prime}}
$$

with constants $a>0$ and $b>0$ independent of $\delta$ and $\epsilon$. For $0<s \leqslant s_{0}$, we have by $(A-g)_{0}-(\mathrm{d})$

$$
\left|h_{t}\left(\delta-i \epsilon+s e^{-i \theta_{i}}\right)\right| \leqslant C
$$

if $\mu_{0} \geqslant 0$ and

$$
\left|h_{t}\left(\delta-i \epsilon+s e^{-i \theta^{\prime}}\right)\right| \leqslant C s^{\mu_{0}}
$$

if $-1<\mu_{0}<0$, where $C>0$ is a constant independent of $\delta$ and $\epsilon$. Therefore, $\left|h_{t}\left(\delta-i \epsilon+s e^{\left.-i \theta_{i}\right)}\right)\right|$ is dominated by an integrable function on $(0, \infty)$ independent of $\delta$ and $\epsilon$. Thus by the Lebesgue dominated convergence theorem we get

$$
\begin{gathered}
\lim _{\epsilon, \delta \vdash 0} \int_{0}^{\infty} h_{t}\left(\delta-i \epsilon+s e^{-i \theta_{t}}\right) e^{-i \theta_{\|}} d s \\
=\int_{0}^{\infty} h_{t}\left(s e^{-i \theta_{i}}\right) e^{-i \theta_{0}} d s
\end{gathered}
$$

Similarly, using $(A-g)_{0}-(\mathrm{b})-(\mathrm{d})$, we can show that $\left|h_{t}(x-i \epsilon)\right|$ is dominated by an integrable function on $(0, \infty)$ independent of $\delta$ and $\epsilon>0$. Thus by the Lebesgue dominated convergence theorem we get

$$
\lim _{\delta \downarrow 0} \lim _{\epsilon \downarrow 0} \int_{\delta}^{\infty} h_{t}(x-i \epsilon) d x=\int_{0}^{\infty} h_{t}(x) d x .
$$

Taking the limit $L \rightarrow \infty$ first, $\epsilon \downarrow 0$ second, and $\delta \downarrow 0$ finally in (2.4) and using (2.5)-(2.7), we obtain

$$
G(t)=G_{1}(t)+G_{2}(t)
$$

with

$$
\begin{aligned}
& G_{1}(t)=\int_{0}^{\infty} h_{t}\left(s e^{-i \theta_{0}}\right) e^{-i \theta_{0}} d s, \\
& G_{2}(t)=-2 \pi i \sum_{a_{k} \in \mathbf{D}_{0 . \theta_{i}}} \operatorname{Res}\left(h_{t}(z), a_{k}\right) .
\end{aligned}
$$

Note that $(A-g)_{0}$ implies that the number of poles of $g(z)$ in $\mathbf{D}_{0, \theta_{0}}$ is finite. Hence the sum with respect to the residue in (2.10) is a finite one and we have

$$
\left|G_{2}(t)\right| \leqslant e^{-a t} P(t)
$$

with a constant $a>0$ and a polynomial $P(t)$ in $t$. On the other hand, by the change of variable $s \rightarrow t^{1 / r} s$, we have 


$$
\begin{aligned}
G_{1}(t)= & \frac{1}{t^{1 / r}} \int_{0}^{\infty} \exp \left[-i\left(s e^{-i \theta_{0}}\right)^{r}\right] g\left(\frac{s e^{-i \theta_{0}}}{t^{1 / r}}\right) e^{-i \theta_{0}} d s \\
= & \frac{1}{t^{\left(\mu_{0}+1\right) / r}} \int_{0}^{\infty} \exp \left[-i\left(s e^{-i \theta_{0}}\right)^{r}\right] \\
& \times\left(s e^{-i \theta_{0}}\right)^{\mu_{0}} u\left(\frac{s e^{-i \theta_{0}}}{t^{1 / r}}\right) e^{-i \theta_{0}} d s
\end{aligned}
$$

where

$$
u(z)=g(z) / z^{\mu_{0}} .
$$

It follows from $(A-g)_{0}-(\mathrm{c})$ and (d) that, for all $s>0$,

$$
\left|u\left(s e^{-i \theta_{10}}\right)\right| \leqslant C /(1+s)^{\mu_{10}+q}<C
$$

with a constant $C>0$ (note that $\mu_{0}+q>\mu_{0}+1>0$ ). Therefore, by the dominated convergence theorem, we get from (2.12)

$$
\lim _{t \rightarrow \infty} t^{\left(\mu_{0}+1\right) / r} G_{1}(t)=g_{0} I\left(\theta_{0}\right)
$$

with

$$
\begin{aligned}
I(\tau)= & \int_{0}^{\infty} \exp \left[-i\left(s e^{-i \tau}\right)^{r}\right] \\
& \times\left(s e^{-i \tau}\right)^{\mu_{i}} e^{-i \tau} d s .
\end{aligned}
$$

The function $z^{\mu_{0}} \exp \left(-i z^{r}\right)$ is analytic in $\{z \in \mathbf{C} \mid-\pi<\arg z<\pi, \quad z \neq 0\}$ and, if $-\pi<r(\arg z)<0$, then it decays exponentially as $|z| \rightarrow \infty$. Therefore, by the Cauchy theorem, we have

$$
\begin{aligned}
I\left(\theta_{0}\right) & =I(\pi / 2 r) \\
& =e^{-i\left(\mu_{0}+1\right) \pi / 2 r} \int_{0}^{\infty} e^{-s^{r} s^{\mu_{0}}} d s \\
& =\frac{e^{-i\left(\mu_{0}+1\right) \pi / 2 r} \Gamma\left(\left(\mu_{0}+1\right) / r\right)}{r} .
\end{aligned}
$$

Combining (2.11), (2.13)-(2.15) with (2.8), we obtain (2.2).

We next consider the case $m>0$. By the change of variable $x^{r} \rightarrow x^{r}-m^{r}$, we have

$$
G(t)=e^{-i t m^{r}} \int_{0}^{\infty} \tilde{g}(x) e^{-i t x^{r}} d x
$$

with

$$
\tilde{g}(x)=\frac{x^{r-1} g\left(\left(x^{r}+m^{r}\right)^{1 / \eta}\right)}{\left(x^{r}+m^{r}\right)^{(r-1) / r}} .
$$

Therefore, the problem is reduced to the case $m=0$; we need only, to check that the function $\tilde{g}(x)$ satisfies $(A-\tilde{g})_{0}$.

Let $\tilde{g}(z)$ be $\tilde{g}(x)$ with $z$ in place of $x$. Then $\tilde{g}(z)$ is meromorphic in $\mathbf{D}_{0, \theta}$ and

$$
\lim _{\epsilon \downarrow 0} \tilde{g}(x-i \epsilon)=\tilde{g}(x), \text { a.e. } x \in(0, \infty) .
$$

Hence $\tilde{g}$ satisfies $(A-\tilde{g})_{0}-($ a $)$.

To prove $(A-\tilde{g})-(\mathrm{b})$, we note that, for $0<a<x<b<\infty$,

$$
\left[(x-i \epsilon)^{r}+m^{r}\right]^{1 / r}=\left(x^{r}+m^{r}\right)^{1 / r}
$$

$$
-\frac{i \epsilon}{\left(x^{r}+m^{r}\right)^{(r-1) / r} x^{1-r}}+O\left(\epsilon^{2}\right)
$$

as $\epsilon \downarrow 0$. Therefore, by the assumption $(A-g)_{m}-(\mathrm{b})$, we get

$$
\sup _{a<x<b}\left|g\left(\left[(x-i \epsilon)^{r}+m^{r}\right]^{1 / r}\right)\right| \leqslant C_{1}
$$

with a constant $C_{1}>0$ independent of $\epsilon$. Further, it is easy to see that

$$
\sup _{a<x<b}\left|\frac{(x-i \epsilon)^{r-1}}{\left[(x-i \epsilon)^{r}+m^{r}\right]^{(r-1) / r}}\right| \leqslant C_{2}
$$

with a constant $C_{2}>0$ independent of $\epsilon$. Therefore we get

$$
\sup _{a<x<b}|\tilde{g}(x-i \epsilon)| \leqslant C_{3}
$$

with a constant $C_{3}>0$ independent of $\epsilon$. Thus $\tilde{g}$ satisfies ( $A-$ $\tilde{g})_{0}-(b)$.

For sufficiently large $|z|$, we have from $(A-g)_{m}-$ (c)

$$
\begin{aligned}
|\tilde{g}(z)| & \leqslant \frac{\text { const }|z|^{r-1}}{\left|z^{r}+m^{r}\right|^{(r-1) / r}\left(z^{r}+m^{r}\right)^{q / r}} \\
& \leqslant \frac{\text { const }}{|z|^{q}} .
\end{aligned}
$$

Therefore $(A-\tilde{g})_{0}-(\mathrm{c})$ follows.

We have

$$
\left(z^{r}+m^{r}\right)^{1 / r} \sim m+m^{1-r} z^{r} / r
$$

as $z \rightarrow 0$. Hence $(A-g)_{m}-(\mathrm{d})$ implies that $\tilde{g}(z)$ has no poles in $\left\{z \in \mathbf{D}_{0, \theta}|| z \mid<\epsilon_{0}\right\}$ with a sufficiently small $\epsilon_{0}$ and

$$
\lim _{z \rightarrow 0} \frac{\tilde{g}(z)}{z^{r\left(\mu_{m}+1\right)-1}}=\frac{g^{m} m^{(1-r)\left(\mu_{m}+1\right)}}{r^{\mu_{m}}} .
$$

Therefore $(A-\tilde{g})_{0}-(\mathrm{d})$ holds with $\tilde{g}_{0}=g_{m}$ $m^{(1-r)\left(\mu_{m}+1\right)} / r^{\mu_{m}}$ and $\mu_{0}=r\left(\mu_{m}+1\right)-1$.

Thus we can apply the result in the case $m=0$ to $\tilde{g}(x)$ and get (2.3).

\section{PROOF OF THEOREM 1.1}

We can write

$$
W(t, f)=\int_{m}^{\infty} g(x) e^{-i t x^{r}} d x
$$

with

$$
g(x)=\frac{f(x) I^{(j)}(x)}{x^{2 r \alpha+j}\left|D_{+}^{(j)}(x)\right|^{2}} .
$$

We shall show that $g$ satisfies $(A-g)_{m}$ in Sec. II. Then Theorem 1.1 follows from an application of Theorem 2.1.

Let

$$
D_{+}^{(j)}(z)=D^{(j)}(z)-2 \pi i I^{(j)}(z), \quad z \in \mathbf{C}_{m},
$$

and define

$$
g(z)=\frac{f(z) I^{(j)}(z)}{z^{2 r \alpha+j} D_{+}^{(j)}(z) D^{(j)}(z)} .
$$

Then, $g(z)$ is meromorphic in $\mathbf{D}_{m, \theta}$. Note that $D_{ \pm}^{(j)}(x)$ is given as

$$
\begin{aligned}
D_{ \pm}^{(j)}(x)= & a_{0}^{(j)}-a_{1}^{(j)} x+\delta_{1, j} \int \rho(\mathbf{k})^{2} d \mathbf{k} \\
& +\mathbf{P} \int \frac{\omega(\mathbf{k})^{j} \rho(\mathbf{k})^{2}}{x-\omega(\mathbf{k})} d \mathbf{k} \\
& +2 \pi i I^{(j)}(x),
\end{aligned}
$$


where $\mathbf{P}$ denotes the principal value. Hence we have

$$
\lim _{\epsilon \downarrow 0} D_{+}^{(j)}(x-i \epsilon)=D_{+}^{(j)}(x), \text { a.e. } x \in(m, \infty) .
$$

Therefore, together with the fact $D_{+}(x)=\overline{D_{-}(x)}$, we get

$$
\lim _{\epsilon \backslash 0} g(x-i \epsilon)=g(x) \text {. }
$$

Thus $g$ satisfies $(A-g)_{m}-($ a $)$.

Property $(A-g)_{m}-(\mathrm{b})$ follows from (AI)(b), (AII) (d) and properties of $f$. $|x|$

By (1.17) and (AII-b), we have for all sufficiently large

$$
\inf _{\epsilon>0}\left|D_{+}^{(j)}(x-i \epsilon)\right| \geqslant C x
$$

with a constant $C>0$. Further, it is easy to see that, for sufficiently large $|z|$ with $\operatorname{Im} z<-\epsilon, z \in \mathbf{D}_{m, \theta}$,

$$
\left|D_{+}^{(j)}(z)\right| \geqslant C_{\epsilon}|z|, \quad\left|D^{(j)}(z)\right| \geqslant C_{\epsilon}|z|
$$

with a constant $C_{\epsilon}$ dependent on $\epsilon>0$. Combining these properties with (AI) (b), (AII) (b), and (f I), we have for sufficiently large $|z|\left(z \in \mathbf{D}_{m, \theta}\right)$

$$
|g(z)| \leqslant \text { const } /|z|^{2 r \alpha+j+q_{j}+2} \text {. }
$$

Since we assume that $2 r \alpha+j+q_{j}+1>0$, we have $2 r \alpha+j+q_{j}+2>1$. Therefore, $(A-g)_{m}-(\mathrm{c})$ follows.

The poles of $g(z)$ come only for the zeros of $D_{+}^{(j)}(z)$ and the poles of $f(z)$. The zeros of $D_{+}^{(j)}(z)$ are discrete and do not accumulate in $\mathbf{D}_{m, \theta}$. It is obvious that $D_{+}^{(j)}(z)$ [resp. $f(z)$ ] has no zeros (resp. poles) in a small neighborhood of $z=m$ in $\mathbf{D}_{m, \theta}\left[\right.$ recall that we assume $d_{m}^{(j)}>0$, see (1.15)] As for the asymptotic behavior of $g(z)$ as $z \rightarrow m$, we have $g(z) \underset{z \rightarrow 0}{\sim} \frac{A_{0}^{(j)} f_{0}}{D_{+}^{(j)}(0) D^{(j)}(0)} z^{-2 r \alpha-j-\alpha_{0}(f)+p_{j}(0)}$

in the case $m=0$ and

$$
g(m+z) \underset{z \rightarrow 0}{\sim} \frac{A_{m}^{(j)} f_{m}}{m^{2 r \alpha+j} D_{+}^{(j)}(m) D^{(j)}(m)} z^{-\alpha_{m}(f)+p_{j}(m)}
$$

in the case $m>0$. Therefore, $g$ satisfies $(A-g)_{m}-(\mathrm{d})$.

Thus we can apply Theorem 2.1 to obtain Theorem 1.1.

\section{AN EXAMPLE}

In this section we consider an example of $\omega$ and see how $p_{j}(m)$ is determined by the dimension $d$ and the infrared property of $\omega$ and $\rho$, where $p_{j}(m)$ is defined by (AII)-(c).

Let

$\omega_{1}(x)=\left(x^{2}+m^{1 / \lambda}\right)^{\lambda}, \quad x \geqslant 0$,

with a parameter $\lambda>0$ (see also Appendix $\mathrm{A}$ ). Then the inverse function $\eta(x)$ of $\omega_{1}(x)$ is given by

$$
\eta(x)=\left(x^{1 / \lambda}-m^{1 / \lambda}\right)^{1 / 2}, \quad x \geqslant m \text {. }
$$

Let $\rho_{1}$ be a continuous function on $[0, \infty)$ satisfying $\rho_{1}(x)>0, \quad x \in(0, \infty)$,

and $\rho$ be given by

$$
\rho(\mathbf{k})=\rho_{1}(|\mathbf{k}|), \quad k \in \mathbf{R}^{d} .
$$

We assume that $\rho_{1}$ has an analytic continuation $\rho_{1}(z)$ onto the domain $\mathbf{D}_{0, \theta_{\rho}}$ with a constant $\theta_{\rho} \in(0, \pi / 2)$ such that

$$
\lim _{\epsilon \backslash 0} \rho_{1}(x-i \epsilon)=\rho_{1}(x), \quad x \in(0, \infty) .
$$

With $\omega_{1}$ and $\rho_{1}$ given above, the function $I^{(j)}(x)$ defined by (1.26) takes the form

$$
I^{(j)}(x)=\frac{V_{d} \eta(x)^{d-2} x^{j+(1-\lambda) / \lambda} \rho_{1}(\eta(x))^{2}}{2 \lambda}, \quad x \in(m, \infty),
$$

where

$$
V_{d}=\int_{S^{d-1}} d S
$$

Lemma 4.1: Assume that, for sufficiently large $|z|$ $\left(z \in \mathbf{D}_{0, \theta_{\rho}}\right)$,

$$
\left|\rho_{1}(z)\right| \leqslant C /|z|^{q(\rho)}
$$

with constants $C>0$ and $q(\rho)>0$ and that

$$
\lim _{z \rightarrow 0} \frac{\rho_{1}(z)}{z^{\gamma(\rho)}}=\rho_{0}
$$

with constants $\gamma(\rho) \geqslant 0$ and $\rho_{0} \neq 0$. Suppose that

$$
q(\rho) / \lambda-j+1-d / 2 \lambda \geqslant 0
$$

and, in the case $m=0$,

$$
(d-2) / 2 \lambda+j+(1-\lambda) / \lambda+\gamma(\rho) / \lambda \geqslant 0 .
$$

Then $I^{(j)}(x)$ satisfies (AII) (a) $-\left(\right.$ c) with $q_{j}$ given by

$$
q_{j}=q(\rho) / \lambda-j+1-d / 2 \lambda
$$

and

$p_{j}(m)=\left\{\begin{array}{l}(d-2) / 2 \lambda+j+(1-\lambda) / \lambda+\gamma(\rho) / \lambda, \\ m=0 \\ (d-2) / 2+\gamma(\rho), \\ m>0\end{array}\right.$

Proof: For a sufficiently small $\theta \in(0, \pi / 2), \eta(z)$ is defined and analytic in $\mathbf{D}_{m, \theta}$ and the image of $\eta$ is included in $\mathbf{D}_{0, \theta_{\rho}}$. Therefore the function

$$
I^{(j)}(z)=\frac{V_{d} \eta(z)^{d-2} z^{j+(1-\lambda) / \lambda} \rho_{1}(\eta(z))^{2}}{2 \lambda}
$$

is analytic in $\mathbf{D}_{m, \theta}$. It is obvious that (AII) (a) holds.

For sufficiently large $|z|\left(z \in \mathbf{D}_{m, \theta}\right)$, we have

$$
C_{1}|z|^{1 / 2 \lambda} \leqslant|\eta(z)| \leqslant C_{2}|z|^{1 / 2 \lambda}
$$

with constants $C_{k}>0, k=1,2$, and hence

$$
\left|I^{(j)}(z)\right| \leqslant \text { const }|z|^{-q_{j}},
$$

with $q_{j}$ given by (4.10). Therefore, (AII)-(b) follows.

Let $m>0$. Then we have

$$
\eta(m+z) \underset{z \rightarrow 0}{\sim}\left(m^{(1-\lambda) / \lambda} / \lambda\right)^{1 / 2} z^{1 / 2}
$$

and hence

$$
\rho_{1}(\eta(m+z)) \underset{z \rightarrow 0}{\sim} \rho_{0}\left(m^{(1-\lambda) / \lambda} / \lambda\right)^{\gamma(\rho) / 2} z^{\gamma(\rho) / 2} .
$$

Therefore we get for $m>0$

$$
\begin{gathered}
I^{(j)}(m+z) \underset{z \rightarrow 0}{\sim} \frac{\rho_{0}^{2} V_{d}}{2 \lambda}\left(m^{(1-\lambda) / \lambda} / \lambda\right)^{\gamma(\rho)+(d-2) / 2} \\
\times m^{j+(1-\lambda) / \lambda} z^{\gamma(\rho)+(d-2) / 2} .
\end{gathered}
$$


In the case $m=0$, we have

$$
I^{(j)}(z) \underset{z \rightarrow 0}{\sim} \frac{\rho_{0}^{2} V_{d}}{2 \lambda} z^{(d-2) / 2 \lambda+j+(1-\lambda) / \lambda+\gamma(\rho) / \lambda} .
$$

Thus (AII)-(c) is satisfied with $p_{j}(m)$ given by Eq. (4.11).

Remark: Condition (AII)(d) follows from the same assumption as in Lemma Bl (see Appendix B).

It is interesting to compare the decay order of $W(t)$ in the case $m=0$ [i.e., $\lambda_{j}$ given by (1.34)] with that in the case $m>0$ [i.e., $\left.p_{j}(m)+1\right]$. By (4.11), we have

$$
p_{j}(0)=p_{j}(m) / \lambda+j+(1-\lambda) / \lambda,
$$

where we take $m>0$.

Hence we get

$$
\begin{aligned}
\Delta_{j} & \equiv \lambda_{j}-\left(p_{j}(m)+1\right) \\
& =((1-\lambda r) / \lambda r)\left(p_{j}(m)+1\right)-2 \alpha .
\end{aligned}
$$

If $\Delta_{j}>0$ (resp. $\Delta_{j}<0$ ), then $W(t)$ with $m=0$ decays faster (resp. slower) than $W(t)$ with $m>0$.

In the models (M1)-(M3), $\Delta_{j}$ is given as follows:

(M1): In this case, we have $r=1, \alpha=j=0$. Hence we get

$\Delta_{0}=((1-\lambda) / \lambda)\left(p_{0}(m)+1\right)$.

(M2): In this case, we have $r=\alpha=\frac{1}{2}, j=0$. Hence we get

$\Delta_{0}=((2-\lambda) / \lambda)\left(p_{0}(m)+1\right)-1$. get

(M3): In this case, we have $r=\alpha=\frac{1}{2}, j=1$. Hence we

$$
\Delta_{1}=((2-\lambda) / \lambda)\left(p_{1}(m)+1\right)-1 .
$$

In the standard case, $\lambda$ is taken as $\lambda=\frac{1}{2}$ (resp. 1,1 ) in the model (M1). [resp. (M2), (M3)]. Therefore, in this case, $W(t)$ with $m=0$ decays faster than $W(t)$ with $m>0$ in each model.

\section{ACKNOWLEDGMENT}

This work was supported in part by the Grant-In-Aid 63740063 and 62460001 for science research from the Ministry of Education, Japan.

\section{APPENDIX A: UNIFORM BOUNDEDNESS OF $\Phi_{\rho}^{(/)}(x-i \epsilon)$}

In this appendix, we consider a sufficient condition for $\Phi_{\rho}^{(j)}(z)$ to satisfy (AI) (a). We define the function $J^{(j)}(x)$ on $\mathbf{R}$ by

$$
J^{(j)}(x)= \begin{cases}I^{(j)}(x), & x>m, \\ 0, & x \leqslant m,\end{cases}
$$

where $I^{(j)}(x)$ is given by $(1.26)$.

Lemma A1: Suppose that $J^{(j)}$ is in $L^{p}(\mathbf{R})$ with some $p \in(1, \infty)$ and satisfies the Lipschitz condition

$$
\left|J^{(j)}(x+h)-J^{(j)}(x)\right|<K|h|^{a}
$$

uniformly in $x$, as $h \rightarrow 0$, with constants $a \in(0,1)$ and $K>0$. Then there exists a constant $C>0$ such that, for all $x \in \mathbf{R}$ and $\epsilon>0$,

$$
\left|\Phi_{\rho}^{(j)}(x-i \epsilon)\right| \leqslant C
$$

Proof: We can write

$$
\Phi_{\rho}^{(j)}(x-i \epsilon)=\left(Q_{\epsilon} * J^{(j)}\right)(x)+i\left(P_{\epsilon} * J^{(j)}\right),
$$

where $P_{\epsilon}$ and $Q_{\epsilon}$ are the Poisson and the conjugate Poisson kernel, respectively,

$$
P_{\epsilon}(x)=\epsilon /\left(x^{2}+\epsilon^{2}\right), \quad Q_{\epsilon}(x)=x /\left(x^{2}+\epsilon^{2}\right),
$$

and * denotes the convolution. Let $\Phi_{\rho}^{(j)}(x)$ be the Hilbert transform of $J^{(j)}$,

$$
\Phi_{\rho}^{(j)}(x)=P \int \frac{J^{(j)}(s)}{x-s} d s .
$$

Then, by the assumption $J^{(j)} \in L^{p}(\mathbf{R})$ and a general theorem [e.g., Ref. 19 (Chap. VI, Lemma 1.5)], we have

$$
Q_{\epsilon} * J^{(j)}=P_{\epsilon} * \Phi_{\rho}^{(j)}
$$

Further, condition (A2) implies that $\Phi_{\rho}^{(j)}(x)$ is continuous in $x \in \mathbf{R}$ with $\Phi_{\rho}^{(j)}(x) \rightarrow 0(|x| \rightarrow \infty)$ and hence, in particular, $\Phi_{\rho}^{(j)}$ is bounded [e.g., Ref. $\left.18(\$ 5.15)\right]$. Similarly, it follows that $J^{(j)}(x)$ is bounded. It is well-known (or easy to prove) that

$$
\left|\left(P_{\epsilon} * f\right)(x)\right| \leqslant \pi|| f \|_{\infty}
$$

for all $f \in L^{\infty}(\mathbf{R})$. Therefore, we get

$$
\begin{aligned}
& \left|\left(Q_{\epsilon} * J^{(j)}\right)(x)\right| \leqslant \pi\left\|\Phi_{\rho}^{(j)}\right\|_{\infty}, \\
& \left|\left(P_{\epsilon} * J^{(j)}\right)(x)\right| \leqslant \pi\left\|J^{(j)}\right\|_{\infty} .
\end{aligned}
$$

From these estimates and (A4), we obtain (A3).

Remark: Condition (A2) implies the continuity of $I^{(j)}(x)$ and hence

$$
\lim _{x \backslash m} I^{(j)}(x)=0
$$
lemma.

A sufficient condition for (A2) to hold is the following

\section{Lemma $A 2$ : Let}

$u(x)=\eta(x)^{d-1} \eta^{\prime}(x), \quad x \in(m, \infty)$,

and $\rho$ be given by

$$
\rho(\mathbf{k})=\rho_{1}(|\mathbf{k}|), \quad k \in \mathbf{R}^{d},
$$

with a continuous function $\rho_{1}$ on $[0, \infty)$. Assume that

$$
\begin{aligned}
& \text { (a) } \lim _{x \downarrow m} u(x)=0, \\
& \text { (b) } \eta(x) \sim C_{\eta} x^{\delta(\eta)}, \\
& u(x) \sim C_{u} x^{\delta(u)} \\
& \rho_{1}(x) \sim C_{\rho} x^{-\delta(p)}
\end{aligned}
$$

as $x \rightarrow \infty$, with strictly positive constants $C_{\eta}, C_{u}, C_{\rho}, \delta(\eta)$, $\delta(u)$, and $\delta(\rho)$.

(c) For all sufficiently small $|h|<1(h \in \mathbf{R})$

$|\eta(x+h)-\eta(x)| \leqslant P_{\eta}(x)|h|^{\epsilon(\eta)}, x \in(m, \infty)$,

$|u(x+h)-u(x)| \leqslant P_{u}(x)|h|^{\epsilon(u)}, \quad x \in(m, \infty)$,

$\left|\rho_{1}(x+h)-\rho_{1}(x)\right| \leqslant P_{\rho}(x)|h|^{\epsilon(\rho)}, \quad x \in[0, \infty)$,

with strictly positive constants $\epsilon(\eta), \epsilon(u), \epsilon(\rho)$, and nonnegative polynomially bounded continuous functions $\boldsymbol{P}_{\eta}$, $P_{u}$, and $P_{\rho}$ on R. Suppose that

$$
\begin{aligned}
& 2 \delta(\eta) \delta(\rho)-\delta(u) \geqslant 0, \\
& \sup _{x>m} P_{u}(x) x^{j-2 \delta(\eta) \delta(\rho)}<\infty,
\end{aligned}
$$




$$
\operatorname{sum}_{x>m} P_{\rho}(\eta(x)) P_{\eta}(x)^{\epsilon(\rho)} x^{j+\delta(u)-\delta(\eta) \delta(\rho)}<\infty .
$$

Then, (A2) holds.

Proof: Let $x \geqslant m$ and $|h|<1$ be sufficiently small. We set $I^{(j)}(m) \equiv 0$. Then we have

$$
\begin{aligned}
J^{(j)}(x+h)-J^{(j)}(x) \\
\quad=I^{(j)}(x+h)-I^{(j)}(x) \\
=V_{d}\left(F_{1}(x, h)+F_{2}(x, h)+F_{3}(x, h)\right),
\end{aligned}
$$

where $V_{d}$ is given by (4.5) and

$$
\begin{aligned}
F_{1}(x, h)= & (u(x+h)-u(x))(x+h)^{j} \rho_{1}(\eta(x))^{2}, \\
F_{2}(x, h)= & \delta_{1, j} u(x) \rho_{1}(\eta(x))^{2} h, \\
F_{3}(x, h)= & u(x+h)(x+h)^{j}\left[\rho_{1}(\eta(x+h))+\rho_{1}(\eta(x))\right] \\
& \times\left[\rho_{1}(\eta(x+h))-\rho_{1}(\eta(x))\right] .
\end{aligned}
$$

(In the case $x=m$, we take $h>0$.) By the assumption, we can estimate as

$$
\begin{aligned}
& \left|F_{1}(x, h)\right| \leqslant \text { const } \frac{\left(x^{j}+1\right) P_{u}(x)|h|^{\epsilon(u)}}{1+x^{2 \delta(\eta) \delta(\rho)}} \\
& \left|F_{2}(x, h)\right| \leqslant \text { const } \frac{x^{\delta(u)}|h|}{1+x^{2 \delta(\eta) \delta(\rho)}} \\
& \left|F_{3}(x, h)\right| \\
& \quad \leqslant \text { const } \frac{(1+x)^{\delta(u)+j} P_{\rho}(\eta(x)) P_{\eta}(x)^{\epsilon(\rho)}|h|^{\epsilon(\eta) \epsilon(\rho)}}{\left(1+x^{\delta(\eta) \delta(\rho)}\right)}
\end{aligned}
$$

These estimates together with (A13)-(A15) imply (A2). In the case $x<m$, (A2) trivially holds.

We conclude this section with an example of $\omega_{1}$ and $\rho_{1}$.

Let $\omega_{1}$ be given by (4.1). We suppose that $\lambda \in(0,1]$. Then, $u(x)$ defined by (A5) takes the form

$$
\begin{aligned}
u(x) & =\frac{x^{(1-\lambda) / \lambda}\left(x^{1 / \lambda}-m^{1 / \lambda}\right)^{(d-2) / 2}}{2 \lambda} \\
& =\frac{x^{(1-\lambda) / \lambda} \eta(x)^{d-2}}{2 \lambda}
\end{aligned}
$$

where $\eta(x)$ is the inverse function of $\omega_{1}$ [see (4.2)]. If $d>2 \lambda$, then we have

$$
\eta(x) \sim x^{1 / 2 \lambda}, \quad u(x) \sim \frac{x^{(d-2 \lambda) / 2 \lambda}}{2 \lambda}
$$

as $x \rightarrow \infty$ and hence (A7) and (A8) hold.

To prove (A10) and (A11), we first note the following elementary estimate.

Lemma A3: Let $\alpha>0$. Then, for all sufficiently small $\epsilon>0$ and $|h|>0$,

$$
\left|(x+h)^{\alpha}-x^{\alpha}\right| \leqslant C\left(1+x^{\alpha-\epsilon}\right)|h|^{\epsilon}, \quad x \geqslant 0,
$$

with a constant $C>0$, where, in the case $x=0$, we take $h>0$.

Proof: It is sufficient to prove (A18) for $h>0$ and $x>0$. Let $\delta \in(0,1)$ be fixed and $x \geqslant h / \delta$. Then we have $h / x \leqslant \delta<1$ and hence

$$
(x+h)^{\alpha}=x^{\alpha}(1+h / x)^{\alpha} \leqslant x^{\alpha}(1+C(h / x))
$$

with a constant $C>0$. Therefore we get

$0<(x+h)^{\alpha}-x^{\alpha} \leqslant C x^{\alpha}(h / x)$

$$
\leqslant C \delta^{1-\epsilon} x^{\alpha-\epsilon} h^{\epsilon}
$$

In the case $x \leqslant h / \delta$, we have

$$
0<(x+h)^{\alpha}-x^{\alpha} \leqslant\left\{(1+1 / \delta)^{\alpha}+1 / \delta^{\alpha}\right\} h^{\alpha} .
$$

Thus (A18) follows.

Lemma A4: Let $\eta(x)$ be the inverse function of $\omega_{1}$ [see (4.2)]. Then, for all sufficiently small $\epsilon>0$ and $|h|$, we have

$$
|\eta(x+h)-\eta(x)| \leqslant \operatorname{const}\left(1+x^{(1 / 2 \lambda)-\epsilon}\right)|h|^{\epsilon}
$$

for the case $m=0$, and

$|\eta(x+h)-\eta(x)| \leqslant$ const $\left[x^{(1-\lambda) / 2 \lambda}\right.$

$$
\left.+\left(x^{1 / \lambda}-m^{1 / \lambda}\right)^{(1 / 2)-\epsilon} x^{(1-\lambda) \epsilon / \lambda}\right]|h|^{\epsilon}
$$

(A20)

for the case $m>0$.

Proof: We first consider the case $m=0$. Then we have $\eta(x+h)-\eta(x)=(x+h)^{1 / 2 \lambda}-x^{1 / 2 \lambda}, \quad x>0$.

Therefore, by Lemma A3, we get (A19).

Let $m>0$ and $m>h>0$. Then we have

$$
\eta(x+h) \leqslant\left\{x^{1 / \lambda}(1+C(h / x))-m^{1 / \lambda}\right\}^{1 / 2}, \quad x \geqslant m,
$$

with a constant $C>0$. Therefore, we get

$0<\eta(x+h)-\eta(x) \leqslant x^{(1-\lambda) / 2 \lambda}\left\{(X+C h)^{1 / 2}-X^{1 / 2}\right\}$

with

$$
X=\left(x^{1 / \lambda}-m^{1 / \lambda}\right) x^{(\lambda-1) / \lambda} .
$$

Therefore, using Lemma A3, we get (A20).

We introduce a function $\theta_{+}(x)$ on $[0, \infty)$ by

$\theta_{+}(x)= \begin{cases}1, & x>0 \\ 0, & x=0\end{cases}$

Lemma A5: Let $u$ be given by (A16).

(a) Let $m=0$ and $d \geqslant 2 \lambda$. Then, for all sufficiently small $\epsilon>0$ and $|h|>0$,

$|u(x+h)-u(x)|$

$\leqslant$ const $\left(1+\theta_{+}(d-2 \lambda) x^{(d / 2 \lambda)-1-\epsilon}\right)|h|^{\epsilon}$, $x \geqslant 0$,

(b) Let $m>0$ and $d \geqslant 2$. Then, for all sufficiently small $\epsilon>0$ and $|h|>0$,

$$
\begin{aligned}
& |u(x+h)-u(x)| \\
& \leqslant \text { const }\left\{1+\theta_{+}(d-2)(x+1)^{[d-2-2 \lambda \epsilon+2(1-\lambda) \epsilon] / 2 \lambda}\right. \\
& \left.\quad+\theta_{+}(1-\lambda) x^{(d-2 \lambda-2 \epsilon) / 2 \lambda}\right\}|h|^{\epsilon}, \quad x \geqslant m . \quad(\mathrm{A} 23)
\end{aligned}
$$

Proof: (a) The case $d=2 \lambda$ is trivial, since we have $u(x)=1 / 2 \lambda$. Let $d>2 \lambda$. Then, we have

$$
\begin{aligned}
u(x+h)-u(x)= & (1 / 2 \lambda)\left\{(x+h)^{(d-2 \lambda) / 2 \lambda}\right. \\
& \left.-x^{(d-2 \lambda) / 2 \lambda}\right\} .
\end{aligned}
$$

Therefore, applying Lemma A3, we get (A.22).

(b) We prove (A.23) only in the case $d>2$ and $0<\lambda<1$. The other cases are easier. We write

$$
\begin{aligned}
u(x+h) & -u(x) \\
= & (1 / 2 \lambda)\left[(x+h)^{(1-\lambda) / \lambda}-x^{(1-\lambda) / \lambda}\right] \eta(x+h)^{d-2} \\
& +(1 / 2 \lambda) x^{(1-\lambda) / \lambda}(\eta(x+h)-\eta(x)) \\
& \times \sum_{i=0}^{d-3} \eta(x+h)^{d-3-i} \eta(x)^{i} .
\end{aligned}
$$


Then, Lemmas A3, A4, (A.19), and (A.20) give (A.23).

Lemmas A4 and A5 show that (A.10) and (A.11) hold.

As for $\rho_{1}$, one can easily find a number of examples which satisfy the conditions required. For example, one can take functions of the form

$$
\rho_{1}(x)=Q(x) /[1+P(x)], \quad x \geqslant 0
$$

where $P(x)$ and $Q(x)$ are polynomials with positive coefficients and $\operatorname{deg} P-\operatorname{deg} Q>0$ is sufficiently large.

\section{APPENDIX B: ESTIMATE FOR $\left|D^{(j)}(x-i \epsilon)\right|$}

In this appendix, we consider a sufficient condition for (AI) (b) to hold.

Lemma B1: Suppose that $\rho(\mathbf{k})>0$ for all $\mathbf{k} \neq 0$. Then, under the assumption in Lemma A1, (AI) (b) holds.

Proof: We have

$$
\begin{aligned}
D_{-}^{(j)}(x)= & a_{0}^{(j)}-a_{1}^{(j)} x+\delta_{1, j} \int \rho(\mathbf{k})^{2} d \mathbf{k} \\
& +\Phi_{\rho}^{(j)}(x)+i \pi I^{(j)}(x) .
\end{aligned}
$$

As stated in the proof of Lemma $A 1, \Phi_{\rho}^{(j)}(x)$ is continuous in $x \in \mathbf{R}$ and

$$
\Phi_{\rho}^{(j)}(x) \rightarrow 0
$$

as $|x| \rightarrow \infty$. Therefore $D_{-}^{(j)}(x)$ is continuous on R. [ For $x<m, D_{-}^{(j)}(x)=D^{(j)}(x)$.] By the Remark after the proof of Lemma Al and condition $d_{m}^{(j)}>0$ [see (1.15)], we have

$$
\inf _{x<m+\delta}\left|D_{-}^{(j)}(x)\right|>0
$$

with some constant $\delta>0$ and hence, taking (1.16) into account, we get

$$
\inf _{\substack{\epsilon \in 0 \\ x \in[m, m+\delta)}}\left|D^{(i)}(x-i \epsilon)\right|>0 .
$$

It follows from (B1) and (B2) that

$$
\left|D_{-(j)}^{(j)}(x)\right| \geqslant \text { const } x
$$

for $x \geqslant R$ with a sufficiently large $R>0$. Combining this estimate with (1.16), we get

$$
\inf _{\substack{\epsilon>0 \\ x>R}}\left|D^{(i)}(x-i \epsilon)\right|>0 .
$$

The positivity of $\rho$ together with that of $\eta$ and $\eta^{\prime}$ implies that

$$
\inf _{\delta<x<R} I^{(j)}(x)>0 .
$$

Hence we have

$$
\inf _{\delta<x<R}\left|D_{-}^{(j)}(x)\right|>0,
$$

which, together with (1.16), gives

$$
\inf _{\substack{\epsilon>0 \\ \delta<x<R}}\left|D^{(j)}(x-i \epsilon)\right|>0 .
$$

Estimates (B3)-(B5) yields (AI) (b).

\section{APPENDIX C: A NECESSARY CONDITION FOR EXPONENTIAL DECAY OF FOURIER TRANSFORMS}

In this section, we give a necessary condition for the Fourier transform $\hat{f}(t)$ of an $L^{2}(\mathbf{R})$ function $f(x)$ to decay exponentially as $t \rightarrow \infty$ and hence a sufficient condition for $f$ under which $\hat{f}$ does not decay exponentially.

Proposition C1: Let $f \neq 0$ be in $L^{2}(\mathbf{R})$ and $\hat{f}(t)$ be the Fourier transform of $f$,

$$
\hat{f}(t)=\frac{1}{\sqrt{2 \pi}} \int_{-\infty}^{\infty} e^{-i t x} f(x) d x, \quad t \in \mathbf{R} .
$$

Suppose that there exist positive constants $C>0$ and $a>0$ such that, for all $t \in \mathbf{R}$,

$$
|\hat{f}(t)| \leqslant C e^{-a|t|}
$$

Then, $\operatorname{supp} f$, the support of $f$, equals the whole real line $\mathbf{R}$.

Proof: [cf. Ref. 15 (\$7.3, Theorem 3.3)] It follows from (C.1) and a general theorem [e.g., Ref. 3 ( $\$ 9.3$, Theorem 9.13) ] that $f(x)$ has an analytic continuation $\tilde{f}(z)$ to the set $S_{a} \equiv\{z|| \operatorname{Im} z \mid<a\}$. Suppose that supp $f \neq \mathbf{R}$. Then, the set $A=\mathbf{R} / \operatorname{supp} f$ is an open set in $\mathbf{R}$ and $f(x)=0$ for all $x \in A$. Hence $\tilde{f}(z)=0$ for all $z \in A$. Therefore, it follows from an elementary property of holomorphic function that $f(z)=0$ for all $z \in S_{a}$. But this contradicts the assumption that $f(x)$ does not vanish identically. Thus supp $f$ must be equal to $\mathbf{R}$.

From Proposition C.1, we have Corollary C2: Let $g \neq 0$ be in $L^{2}(\mathbf{R})$ and put

$$
G_{a, b}(t)=\int_{a}^{b} e^{-i t x} g(x) d x .
$$

Suppose that $-\infty \leqslant a<b<\infty$ or $-\infty<a<b \leqslant \infty$. Then, $G_{a, b}(t)$ does not decay exponentially as $|t| \rightarrow \infty$.

Proof: We need only to apply Proposition C1 with $f=\sqrt{2 \pi} \chi_{[a, b]} g$, where $\chi_{[a, b]}$ is the characteristic function on $[a, b]: \chi_{[a, b]}(x)=1, x \in[a, b] ; \chi_{[a, b]}(x)=0, x \notin[a, b]$.

${ }^{1}$ A. Arai, "Perturbation of embedded eigenvalues in Fock spaces: A general class of exactly soluble models." Hokkaido Univ. Preprint Series in Math. No. 21, February, 1988.

${ }^{2}$ M. Reed and B. Simon, Methods of Modern Mathematical Physics, Vol. I: Functional Analysis (Academic, New York, 1972).

${ }^{3}$ M. Reed and B. Simon, Methods of Modern Mathematical Physics, Vol. II; Fourier Analysis, Self-Adjointness (Academic, New York, 1975).

${ }^{4} \mathbf{K}$. Lindenberg and B. J. West, "Statistical properties of quantum systems: The linear oscillator," Phys. Rev. A 30, 568 (1984).

${ }^{5} \mathrm{E}$. Braun, "Irreversible behavior of a quantum harmonic oscillator coupled to a heat bath." Physica A 129, 262 (1985).

${ }^{\circ} \mathrm{E}$. Braun and P. A. Mello, "The correlation function for a quantum oscillator in a low-temperature heat bath," Physica A 143, 547 (1987).

${ }^{7}$ A. Arai, "Spectral analysis of a quantum harmonic oscillator coupled to infinitely many scalar bosons," J. Math. Anal. Appl. 139 (1989).

${ }^{8} \mathrm{~F}$. Schwabl and W. Thirring, "Quantum theory of laser radiation," Ergeb. Exact. Naturwiss. 36, 219 (1964).

${ }^{9}$ A. Arai, "On a model of a harmonic oscillator coupled to a quantized, massless, scalar field. I," J. Math. Phys. 22, 2539 (1981).

${ }^{10} \mathrm{~A}$. Arai, "On a model of a harmonic oscillator coupled to a quantized, massless, scalar field. II," J. Math. Phys. 22, 2549 ( 1981 )

"H. Maassen, "A quantum field acting as a heat bath," Phys. Lett. A 91 , 107 (1982).

${ }^{12} \mathrm{C}$. Aslangul, N. Pittier, and D. Saint-James, "Time behavior of the correlation functions in a simple dissipative model," J. Stat. Phys. 40,167 (1985).

${ }^{13} \mathrm{P}$. Schramm and H. Grabert, "Low-temperature and long-time anomalies of a damped quantum particle," J. Stat. Phys. 49, 767 (1987). 
${ }^{14}$ A. Arai, "Rigorous theory of spectra and radiation for a model in quantum electrodynamics," J. Math. Phys. 24, 1896 (1983).

${ }^{15}$ E. B. Davies, Quantum Theory of Open Systems (Academic, London, 1976).

${ }^{16} \mathrm{H}$. Grabert, U. Weiss, and P. Talkner, "Quantum theory of the damped harmonic oscillator," Z. Phys. B 55, 87 (1984)
${ }^{17} \mathbf{R}$. Jung, G. L. Ingold, and H. Grabert, "Long-time tails in quantum Brownian motion," Phys. Rev. A 32, 2510 (1985).

${ }^{18}$ E. C. Titchmarsh, Theory of Fourier Integrals, 2nd ed. (Oxford, U. P., Oxford, 1948).

${ }^{19}$ E. M. Stein and G. Weiss, Fourier Analysis on Euclidean Spaces (Princeton U.P., Princeton, NJ, 1971). 
Journal of Mathematical Physics is copyrighted by the American Institute of Physics (AIP). Redistribution of journal material is subject to the AIP online journal license and/or AIP copyright. For more information, see http://ojps.aip.org/jmp/jmpcr.jsp Copyright of Journal of Mathematical Physics is the property of American Institute of Physics and its content may not be copied or emailed to multiple sites or posted to a listserv without the copyright holder's express written permission. However, users may print, download, or email articles for individual use. 